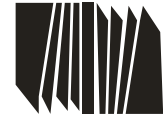

UPE L

UNIVERSIDAD PEDAGOGICA EXPERIMENTAL LIBERTADOR

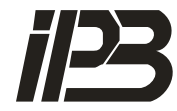

INSTITUTO PEDAGOGICO DE BARQUSIMETO

LUIS BELTRAN PTRO FIGUEROA

\section{REVISTA}

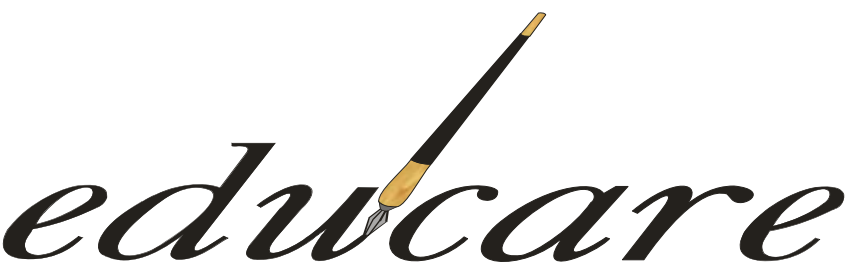

Órgano Divulgativo de la Subdirección de Investigación y Postgrado del Instituto Pedagógico de Barquisimeto "Luis Beltrán Prieto

Figueroa"

BARQUISIMETO - EDO. LARA - VENEZUELA

NUEVA ETAPA

VERSION ELECTRÒNICA

DEPOSITO LEGAL: ppi201002LA3674

Volumen $14 \mathrm{~N}^{\circ} 1$

ISSN: 2244-7296

\title{
LA ESCUELA COMO CENTRO PROMOTOR DE SALUD
}

\section{THE SCHOOL AS HEALTH SPONSOR}

\section{Rafael Gaspieri Romero}

(UCLA) 


\title{
LA ESCUELA COMO CENTRO PROMOTOR DE SALUD
}

\author{
THE SCHOOL AS HEALTH SPONSOR
}

\section{INVESTIGACIÓN}

Recibido:12-03-09

\section{Rafael Gaspieri Romero*}

UCLA

Aceptado: 24-02-10

\section{RESUMEN}

El presente trabajo es producto de una investigación de tipo cualitativo que se realizó con el objetivo de evaluar los logros del proyecto de Extensión Escuelas Promotoras de Salud. Se ejecutó en cinco escuelas de los municipios Iribarren y Palavecino del Edo. Lara . El enfoque onto-epistemológico fue Hermenéutico y eminentemente interpretativo. Se empleo como técnica de recolección de información los grupos focales aplicada a un total de 79 docentes. Entre los logros más destacados del proyecto se tienen: influencia positiva en el ambiente organizacional de las escuelas, incremento de la participación de los docentes y comunidad educativa, así como el aprendizaje y utilización de conceptos nuevos en el área de la salud. La confluencia entre salud y educación que se encontró en este trabajo confirma que ambas sectores comparten las aspiraciones y objetivos de desarrollo humano, contemplados en las metas del milenio de la Organización Mundial de la Salud.

Descriptores: Escuelas, promoción de la salud, evaluación cualitativa.

\begin{abstract}
The present work is the product of a qualitative study which had the goal of evaluating the achievements in a project called Extension Health Promoting Schools. It was applied in five schools of Iribarren and Palavecino townships at Edo. Lara . The onto-epistemological approach was hermeneutic and highly interpretive. The focus group was information gathering, a technique applied to a total of 79 teachers. Among the outstanding achievements of the project, it was found, a positive influence on the organizational school environment, increased participation of teachers and the education community as well as a learning and practice of new concepts on health area. The convergence between health and education that occurred in this study confirms that both fields share hopes and goals of human development proposed by the World Health Organization as 21st century goals.
\end{abstract}

Keywords: Schools, health promotion, evaluative research

\footnotetext{
* Universidad Centroccidental Lisandro Alvarado. Decanato de Ciencias de la Salud. Docente Titular, Departamento de Medicina Preventiva y Social. Barquisimeto. Edo Lara . Venezuela.

rgasperi@ucla.edu.ve,rgasperir@yahoo.com.ve
} 


\section{INTRODUCCIÓN}

La salud y el bienestar son conceptos sociales y poblacionales, más que individuales. En Iberoamérica cada año, se presentan numerosas muertes, como consecuencia de afecciones que hubieran podido prevenirse, las que pueden agruparse en tres grandes categorías:

1 ) Muertes debidas a enfermedades prevenibles mediante vacunación oportuna o como consecuencia de desnutrición, condiciones de vida inadecuadas, falta de agua potable y ausencia de sistemas para la eliminación de excretas.

2 ) Muertes ocasionadas por afecciones que podrían haberse tratado con éxito mediante el acceso oportuno a servicios de salud de buena calidad.

3 ) Muertes ocasionadas por violencia, ya sea por acción directa (homicidios) o negligencia (accidentes).

Por otra parte, es importante aclarar, que pese a que se han logrado avances importantes en la disminución de la desnutrición infantil en América Latina y el Caribe, todavía hay tasas altas de deficiencia de micro nutrientes y desnutrición crónica, especialmente en algunos países con población numerosa y tasas elevadas de mortalidad infantil, Organización Panamericana de la Salud (OPS) 2007

Se calcula que en el año 2004 cerca de 36\% de los menores de 2 años de la Región se encontraban en situación de alto riesgo desde el punto de vista nutricional, en especial en las zonas rurales, en donde la proporción fue incluso mayor (46\%). Una consecuencia típica de la desnutrición es que, a través de variables neuro psicológicas intermedias, afecta considerablemente el rendimiento escolar. Adicionalmente, los niños desnutridos no asisten al colegio con regularidad o lo abandonan por completo y repiten años con frecuencia. En consecuencia, la desnutrición infantil es una de las causas principales de disminución de la calidad del producto, de los programas educativos. En comunidades donde hay grandes desigualdades sociales y económicas entre los individuos, los niveles de salud y bienestar (físico, cognitivo y psicosocial) son inferiores que en las comunidades en donde las diferencias son menores, lo que destaca el papel fundamental que desempeña la falta de equidad de bienes y servicios, como factor determinante en la salud de la población. 
Pobreza, exclusión, vulnerabilidad social, falta de oportunidades, trabajo infantil, violencia intrafamiliar y violencia sociopolítica (incluido el desplazamiento forzado) son otros factores que amenazan la vida y el desarrollo saludable de miles de niños, niñas y jóvenes en la Región, en donde más de la mitad viven en la pobreza. OPS (2007).

Venezuela no escapa a este panorama. Nuestra población infantil, sobre todo la escolar que representa un 23\% de la población general, resulta afectada con problemas como las altas cifras de fracaso académico, deserción escolar y el alto índice de niños fuera del sistema educativo. A lo anterior se suman factores que contribuye a agravar la situación de este grupo etario, como es su estado de salud, encontrándose, según el Ministerio del poder popular para la Salud, MPPS, (2002) un alto porcentaje de desnutrición, problemas infecciosos, parasitarios, defectos visuales y auditivos que dificultan el rendimiento escolar y su éxito académico.

Los servicios de salud escolar del MPPS, sólo cubren un 22\% de esta población y las escuelas no cuentan con la dotación suficiente ni la preparación de los docentes para solventar estos problemas, Gasperi (1998). Esta situación deja a este grupo de población en un estado de vulnerabilidad tal, que puede afectar el desarrollo y crecimiento físico, mental, social y espiritual, de casi una cuarta parte de nuestra población, dejando como consecuencia un futuro incierto para nuestro país.

En tal sentido, surge una estrategia viable para ayudar a solventar parte de los problemas antes citados, como es la creación de las redes de Escuelas Promotoras de Salud, cuya intención es promover una mejor calidad de vida de los alumnos en el ámbito de su escuela y que trascienda a su entorno y mejore en consecuencia su rendimiento académico y su futuro desempeño como ciudadano.

Una Escuela Promotora de Salud es aquella que proporciona condiciones óptimas para el desarrollo emocional, intelectual, físico y social de los niños. En ella se trata de promover, fomentar y permitir la adquisición de habilidades personales y sociales que conduzcan a crear valores y actitudes positivos hacia la salud, como: la capacidad en la toma de decisiones personales, la participación, el autocuidado de su salud, la solidaridad, cooperación e igualdad, elementos que deben estar acordes al desarrollo físico, mental y sociocultural del niño, OPS,(2003). El papel del docente es favorecer y permitir el desarrollo de estas habilidades: la escuela y el Ambiente Organizacional de la institución tienen gran importancia en la formación integral del estudiante. 
Hay pruebas abundantes que confirman los beneficios que se derivan de la cooperación entre Salud y Educación, por ejemplo se puede decir que en los hogares constituidos por personas con mayor grado de educación, el estado de salud y la calidad de vida, tanto de niños como de adultos es mejor y además tienen consecuentemente más posibilidad de acceso a recursos, bienes y servicios OPS, (1995)

El presente trabajo presenta los resultados de una experiencia que da cuenta de la responsabilidad social del sector salud y educación en especial de la Universidad, a través de su proyecto de Extensión Universitaria Escuelas Saludables; pero evaluada y estudiada bajo otra óptica, en la cual, no sólo se genera información cuantificable de los hechos, sus porcentajes y tasas, sino que permitan responder preguntas “cómo”, “por qué” se sucedieron los hechos. Así mismo permite analizar “qué se aprendió” en el proceso y que además aporte datos confiables para la toma de decisiones a los gerentes de proyectos sociales.

El objetivo general de esta investigación es: Identificar y analizar los logros alcanzados por el proyecto "Escuelas Saludables" mediante una investigación evaluativa de tipo cualitativo.

\section{CONSIDERACIONES TEÓRICO}

Desde 1978, cuando se promulga en Alma-Ata (ex Unión Soviética) la Declaración de Atención Primaria de Salud, como una estrategia para mejorar la salud de todos los pueblos del mundo y lograr la meta de salud para todos en el año 2000, se sugirió la promoción de la salud, como una vía para hacer realidad esta meta. En la década de los ochenta, se le da cada vez más importancia a la promoción de la salud en las políticas sanitarias de los gobiernos y se exige como un pilar en las acciones gubernamentales de muchos países desarrollados como Canadá, España, Italia y demás países en vías de desarrollo como Chile, Brasil, Cuba, entre otros. Cobra tanto auge el concepto de Promoción de la Salud, que en 1986 se celebra en Ottawa, una reunión en donde se suscribe la Carta de Ottawa, y se redimensiona el concepto de Salud, expresando:

La salud se crea y se vive en el marco de la vida cotidiana: en los centros de enseñanza, de trabajo y de recreo. La salud es el resultado de los cuidados que uno se dispensa a sí mismo y a los demás, de la capacidad de tomar decisiones y controlar la vida propia y de asegurar que la sociedad en que 
uno vive ofrezca a todos sus miembros la posibilidad de gozar de un buen estado de salud.

De igual forma se plantean requisitos para alcanzar la salud, que están en función de determinantes externos como: la paz, educación, vivienda, alimentación, empleo decente, ambiente sano, justicia social y equidad. Igualmente determinantes internos como: las creencias, convicciones, formación, inteligencia y emociones.

A partir de estas conceptualizaciones, se redimensiona la concepción de salud más como un proceso, que como un producto. En términos prácticos, es calidad de vida, en donde se les brinda a los ciudadanos, conscientemente, los medios y la certidumbre que les hace sentirse dueños de sus propias existencias, y por lo tanto la salud se puede construir y conseguir desde nuestros ámbitos de acción, como la escuela por ejemplo, no obviando en ese proceso la responsabilidad que tiene el Estado y los sistemas de salud en esa misión.

De igual forma, se continuaron con reuniones mundiales y regionales para discutir sobre el mismo tema. En 1988 en Adelaida (Australia), y en la década de los 90 en Sundsval (Suecia), en 1991 y Santa Fe de Bogotá (Colombia), en 1992, y la Carta del Caribe de Promoción de la Salud en 1993. Finalmente, al término del siglo XX, en Yakarta, Indonesia, se declara que la promoción de la salud actúa sobre los factores determinantes de la salud, para derivar el máximo beneficio posible a la población, disminuir la inequidad, garantizar el respeto a los derechos humanos y acumular capital social, OPS (2002)

Las estrategias de la promoción de la salud se plantean en cinco campos de acción como:1) Desarrollar políticas públicas favorables a la salud, para darle viabilidad política y legislativa. 2 ) Crear entornos favorables a la salud con la intención de facilitar el aprendizaje de comportamientos que permitan la protección del entorno y la conservación de los recursos naturales, para la convivencia armónica hombre-entorno. 3) Reforzar la acción comunitaria, facilitando así la integración cada vez mayor de las personas en su autocuidado y capacitándolas para que asuman su destino y el de sus familias y comunidades. 4) Reorientación de los servicios de salud, dándole mayor énfasis a la prevención y promoción de la salud y guiar más las acciones hacia la salud, que hacia la enfermedad. 5) Desarrollar las actitudes, aptitudes y los recursos individuales, ya que la salud es una construcción personal en la que intervienen 
creencias, expectativas, cultura, condiciones socioeconómicas y una forma muy particular de concebir la vida. El proyecto Escuelas Saludables, que es la experiencia que aquí analiza sus logros, basa sus acciones en estas estrategias, con especial énfasis en las dos ultimas, es decir la participación social y el desarrollo de aptitudes y actitudes hacia una nueva concepción de salud, materializándose esta estrategia, a través de la educación para la salud.

Las Escuelas Saludables son definidas por la OPS, OMS ( 2003) como una iniciativa para desarrollar un conjunto de acciones dirigidas a la producción social de salud en el ámbito escolar, facilitando la adopción por toda la comunidad educativa de estilos de vida sanos, en ambientes favorables a la salud.

$\mathrm{Su}$ objetivo fundamental es formar futuras generaciones que dispongan de conocimientos, habilidades y destrezas necesarias para promover y cuidar su salud, la de su familia y su comunidad, así como crear y mantener ambientes de estudio, trabajo y convivencias saludables. Está fundamentada en la moderna concepción de salud, definida en la Carta de Ottawa en 1986.

Conceptualmente la promoción de la salud, así como la educación para la salud, que es su estrategia fundamental, se nutre de una enorme diversidad de teorías y modelos que tienen fundamento en la investigación científica originada en distintas disciplinas y latitudes, especialmente en los Estados Unidos de Norteamérica y Europa, con excepciones notables como la investigación en el campo de la acción participativa (Fals Borda 1988-91) y la teoría de la educación participativa (Freire 1970-74), que se desarrollaron en América Latina, Ippólito S (2003). A continuación se sintetiza algunas de estas, que sustentan la promoción y la educación para la Salud, y en las de una u otra forma se fundamenta, las estrategias del proyecto, Escuelas saludables.

Teorías sobre desarrollo cognitivo centrado en quien aprende

Muchos de los pilares actuales sobre desarrollo cognitivo se deben al trabajo pionero de Jean Piaget, quien en la década de los treinta postuló que el desarrollo cognitivo en los seres humanos progresa a lo largo de una serie de etapas (sensoriomotriz, preoperacional, de operaciones concretas y de operaciones formales), como resultado de la interacción permanente entre los factores biológicos y la experiencia, y que en cada una de ellas la persona adquiere la capacidad de comprender el mundo de manera cada vez más compleja y sofisticada, Lejarraga,H(2004). Los marcos de referencia de 
desarrollo cognitivo centrado en quien aprende, que se fundamentan principalmente en las teorías de educación de adultos, Knowles 1981-1984, pedagogía crítica, Freire 1970-1974, y aprendizaje social, Bandura 1986, Hipólito, S (2003). En ese orden de ideas, se prioriza en el proyecto en la importancia de centrar la educación en el alumno, tomar en cuento sus necesidades, no solo académicas, sino físicas- biológicas, sociales y emocionales. Igualmente el comprender al niño como un ser en crecimiento y desarrollo, especialmente las etapas de la vida con las que trabajamos, edades preescolar y escolar, los niños adquieren las bases de su comportamiento y conocimientos, su sentido de responsabilidad y la capacidad de observar, pensar y actuar. De igual forma, es en esta etapa cuando el niño adopta hábitos higiénicos que durarán toda su vida, descubre la potencialidad de su cuerpo y desarrolla habilidades y destrezas para cuidar su salud y colaborar con el cuidado de su familia y la comunidad.

\section{El Modelo de Creencias en Salud}

Se formuló en la década de los cincuenta a partir de una experiencia de participación pública en un programa de tamizaje (screening) para la tuberculosis. Se basa en el análisis de las distintas fuerzas y factores que influyeron en la participación de las personas en los tratamientos, Greene,G (1988).Pone de relieve las creencias, sobre el fenómeno Salud enfermedad y las información que se tenga sobre este, producto de su proceso de socialización y los elementos culturales donde se desenvuelva el individuo, esto generara una actitud positiva o negativa a actuar sobre determinado tratamiento y en consecuencia generara o no, la conducta deseada de actuación sobre determinada enfermedad, OPS (2006). A lo largo de la ejecución del proyecto, se trabajo con los docentes, ideas y creencias que estos poseían sobre salud, para incorporar otros nuevos conceptos mas acordes con el ideario de la escuela como promotora de salud.

\section{La Teoría del Aprendizaje Social}

Propuesta por Bandura sugiere que las personas se comprometen en la modificación del comportamiento siempre y cuando, consideren que son competentes para adoptar el nuevo comportamiento, es decir, se perciben a sí mismas como individuos eficientes, Alvarado, J (1996). Además incorpora conceptos como la necesidad de poseer conocimiento y destrezas para realizar una conducta, la importancia de las expectativas para obtener resultados anticipados de la conducta y el aprendizaje por observación. Así 
mismo habla del determinismo reciproco como la interacción dinámica entre una persona, su conducta y el ambiente donde se desenvuelven .OPS(2006) En este sentido, siempre se insistió con el docente en las implicaciones de todos estos preceptos en el desarrollo del proyecto en las escuela y con sus alumnos como por ejemplo: aprendizajes a través de practicas de destrezas, modelar resultados positivos de una conducta saludable, incluir modelos de roles con credibilidad para la conducta buscada y considerar múltiples caminos para el cambio conductual, incluyendo cambios ambientales, personales y destrezas particulares.

Las teorías y modelos de la persuasión

Worchel, S (2002) ofrecen un marco de referencia amplio para la comprensión del comportamiento humano y sus determinantes, han servido como base para la investigación científica en disciplinas relacionadas con las ciencias de la salud y la planificación, implementación y evaluación de actividades de promoción para la salud. Se debe persuadir a los docentes y estos, al resto de la comunidad educativa con las bondades del proyecto, con los materiales instruccionales, con el poder de la comunicación y convencimiento honesto y transparente.

Diversas experiencias en la región son alentadoras e ilustra como es posible avanzar en las Metas del milenio para el Desarrollo, adoptadas por países adscritos a la ONU, mediante estrategias integradoras como la iniciativa regional Escuelas promotoras de salud, OPS(2002),OPS(2006),OPS(2007). Dicha iniciativa es producto de varios años de consenso y se desarrollo con base en las experiencias de los países con el fin de alcanzar un marco integrador de las acciones de salud escolar. En los países donde se ha evaluado, los resultados muestran que la iniciativa es exitosa, produce mejoría en los ambientes escolares, en la calidad y los resultados de la educación para la salud, y en general es bien calificada por los profesores y gerentes del sector educativo. OPS (2003)

Por esto, es preciso que se formen escuelas y docentes proactivos, eficientes, innovadores y creativos que cumplan con su función social y en donde se conciba a la salud, como el eje del proceso organizacional, para lograr la excelencia académica, a través del mejoramiento de la calidad de vida de sus alumnos. 


\section{EL PROCESO DE LA INVESTIGACIÓN}

Este trabajo se inscribe en el paradigma cualitativo, justificado en el hecho de que su objeto de estudio, es la evaluación de un proyecto de gran contenido social, en donde existe la necesidad de interpretar desde la vivencia de los actores una experiencia en promoción de salud, cuya realidad compleja, múltiple y relativa debe ser reconstruida y analizada no desde la mera descripción o cuantificación del fenómeno exterior o aparente, sino desde el interior, de lo no aparente, de lo que emerge y de los significados que la experiencia representa para sus protagonistas.

Esta investigación, centra su análisis en valorar como se dio el desarrollo del proyecto de Extensión Escuelas Saludables, además de evidenciar, que se ha logrado y que se ha aprendido. Es aquí donde el método cualitativo aporta estrategias que permiten responder de forma más amplia, detallada y clara estas preguntas que los métodos de evaluación tradicional. De igual forma, nos permite descubrir las realidades y simultáneamente ordenarlas analíticamente, teniendo en cuenta elementos metodológicos, contextuales y relacionales.

1) Enfoque onto-epistemológico del método.

Dentro del paradigma cualitativo o naturalista, el interpretativismo es la corriente epistemológica que más se adapta a la presente investigación por todo lo expresado anteriormente, y dentro de esta tendencia la hermenéutica es el enfoque epistemológico adoptado en este trabajo, ya trata de comprender textos y fenómenos socio-educativos, se puede conocer el pensamiento humano con la ayuda de símbolos y palabras analizados e interpretados en su contexto y realidad socio-histórica ( Valles,1999,pp.7577). siendo esto de gran ayuda a la hora de valorar adecuadamente un resultado o una actividad en el marco de un proyecto social.

2 ) El escenario de la investigación

a) Contexto

La investigación se desarrolla en el marco del proyecto Escuela Saludable en el Municipio Iribarren del Estado Lara, Venezuela, donde se ejecutan las actividades en cinco escuelas ubicadas en diferentes barrios humildes de la ciudad, desde el 2002 al 2006, cuando se recoge la información.

El mencionado proyecto se ejecutó en tres etapas: la primera contemplaba la selección de las escuelas, la presentación y negociación con el personal para desarrollar 
el proyecto, así como la implementación del plan de capacitación al personal docente y representantes de la comunidad educativa, mediante las realizaciones de ocho talleres. La segunda etapa era la aplicación de los conocimientos adquiridos en un plan de acción (de plantel y de aula particulares). En estas escuelas, una vez culminado el plan de capacitación, cada una diseñó su plan de acción que fue aplicado durante el siguiente año escolar, y estuvo a cargo de la comisión de salud escolar de cada institución, con los docentes y directivos planificando y ejecutando dichas actividades. La tercera etapa, monitoreo y evaluación del impacto.

En el momento de planificar este trabajo de investigación, el proyecto debía realizar un monitoreo de las actividades contempladas en su tercera etapa, surgió en el seno del equipo planificador la necesidad de hacer un seguimiento de los procesos y valorar los resultados parciales o intermedios y que a su vez esto sirviera para ser presentado ante las autoridades competentes $y$ entes financieros para darle sostenibilidad económica al proyecto

b) Población y sujetos de estudio

El universo que cubre las cinco escuelas abordadas por el proyecto son 5.800.000 alumnos aproximadamente y 160 docentes con un alcance indirecto a 1200 familias. Todas las escuelas tenían en común las siguientes características: (1) manejaban una matrícula que abarcaba la primera y segunda etapa de educación básica, de 1ero a 6to grado, población escolar a la cual se dirigió el proyecto; (2) mantener estrecho contacto con un ambulatorio del Ministerio de Salud y de ser posible, que éstos formen parte de la red de ambulatorios donde la Facultad de Medicina hace sus pasantías; (3) todos debían pertenecer al Municipio Iribarren; (4) las características de las escuelas deberían ser "término medio", es decir no ser escuelas de primera calidad con excelentes condiciones de infraestructura, dotación y nivel educativo, donde no hiciera falta todo el esfuerzo físico y económico del proyecto, ni escuelas con tantos problemas de infraestructura, dotación y nivel educativo, donde el proyecto no pueda resolver esos inconvenientes de orden estructural y crear falsas expectativas en la comunidad educativa y frustración en el equipo. Los sujetos a estudiar fueron 79 docentes, constituidos en 5 grupos focales de todas las escuelas que participaron en el proyecto.

3) Técnicas, instrumentos y procedimientos

Se empleó la técnica de grupos focales y los testimonios focalizados, técnica que tiene como base el principio de la complementariedad propuesta por Neil Bohr, como 
aspecto central de la descripción de la naturaleza y sus fenómenos (Martínez, 2004, pp.2002 ). Una vez solicitado el permiso respectivo en cada escuela, se concertó la cita para la realización de la reunión del grupo focal, con los docentes. Los sujetos de estudio fueron seleccionados de acuerdo a su participación en el proyecto desde el inicio y asistido a todos los talleres o estar participando en ese momento, aunque no hubieran asistido a todos los talleres.

La reunión fue previamente preparada, día, hora, lugar, y sirvió como instrumento un guión de preguntas preparado para tal fin, contentivo de cinco preguntas y grabada en reproductor tipo periodista., y luego éstas fueron transcritas en el procesador de palabras Microsoft Word, para su posterior análisis.

La sesión de trabajo estuvo conformada, además del investigador y el grupo, por un asistente al investigador, quien anotaba los aspectos más importantes de las entrevistas y aquellos temas o pasajes donde hubiere mayor controversia o exteriorización de emotividad. Al inicio de ésta se daban las instrucciones para el adecuado desarrollo de la sesión de trabajo y se les informaba de la finalidad del trabajo que se estaba realizando y de la importancia de sus aportes para el éxito de la investigación. El investigador hacía intervenciones puntuales, sobre todo cuando era necesario aclarar una idea importante, evitar redundancias excesivas (al agotarse el discurso) o mucha dispersión del tema por cierto tiempo. Al final de la entrevista se hacía un cierre general sobre la actividad y se le daba libertad a cualquier miembro del grupo a emitir una opinión o comentario, que pudiera aportar al tema.

Se utilizó igualmente además como estrategias de apoyo a los grupos focales, otras técnicas e instrumentos con el propósito de obtener información confiable y complementaria que contribuyera a una mayor comprensión de la realidad a evaluar y como prueba de validez de los resultados obtenidos. Se empleó un cuestionario semiestructurado, para caracterizar a los sujetos de estudio según variables demográficas, además de la revisión de documentos, estadísticas y otras fuentes.

Para el análisis de la información obtenida en los grupos focales, se empleó el programa denominado Atlas-ti, desarrollado por la Universidad de Berlín en 1994, para el procesamiento de información cualitativa asistido por computadoras, basándose en los principios de la teoría fundamentada de Glasser y Strauss ( 1997 ) y analizándose por separado las transcripciones de cada grupo focal en Unidades hermenéuticas, empleando lo que Martínez (2004), denomina propedéutica fenomenológica, 
conformada por los siguientes pasos: codificación - categorización - estructuración contrastación - conformación de redes conceptuales - teorización.

\section{RESULTADOS Y DISCUSIÓN}

Los resultados de la investigación evaluativa realizada, muestran los logros del proyecto de Extensión Escuelas Saludables, relacionados con siete categorías, donde este impactó con mayor intensidad en la vida institucional y del personal docente. Estos datos se evidenciaron en la red conceptual que emergió del discurso de los grupos focales con apoyo del Software ATALS TI. A continuación se describen los resultados encontrados en las respectivas categorías: Ambiente organizacional, participación del docente, aprendizajes de conceptos relacionados con el proyecto, participación de la comunidad educativa, utilidad en la salud del niño, prestigio institucional y mejor integración con el entorno, beneficio personal y laboral del docente.

\section{- Ambiente Organizacional.}

La motivación y el interés de los docentes y niños en las actividades del proyecto, evidenciado por la participación del docente, alumnos y representantes identificados con el proyecto, ha contribuido a consolidar el ambiente organizacional, enalteciendo valores como el compromiso, la participación, la cooperación y la vida sana. De igual forma con el taller de Gerencia, impartido a los docentes y directivos se construyo la misión, la visión compartida por toda la comunidad educativa y se reflexiono la razón de ser de las escuelas y sus docentes, influyendo esto positivamente en el ambiente organizacional de la institución. Se citan a continuación, algunos fragmentos de los grupos focales que ilustran tales aseveraciones.

...observamos que hay como bastante motivación de parte de todos los entes involucrados en este proceso, comunidad educativa, docentes, los mismos alumnos interesados en las actividades que se están realizando (Preg. 1 1:9; 78:81) ....la parte de salud está dentro de nuestra visión, eso fue modificado, fue rectificado, lo que es la misión y visión y ya está involucrada la parte de salud...” (Preg1 1:60; 753:755).

En otra escuela:

...Una gran receptividad por parte de la mayoría de los docentes, hemos sido multiplicadores, muchos de nosotros, en eso, y se ha notado muchísimo al incorporar a los padres ... (Preg.1 1:31). ...hubo una motivación que las 
personas, incluso los que no asistieron buscaban esta orientación, mira que dieron, y uno estaba pendiente de la guía, los temas, los ejercicios....

En otra escuela se comenta:

...docentes que se incorporaron en este año pero ve que hay en el ambiente esa visión, esa motivación, esa incentivación, pues se han ido involucrando, que han llegado a la institución, por traslado y entonces se han ido involucrando, y se han ido motivando, están ya sensibilizados a seguir en la misa tónica de querer ver a sus representantes la parte de salud... (Preg.1.1:30; 243:249)

El ambiente organizacional como se recordara, es un termino genérico que hace referencia a las percepciones que los docentes tienen de su ambiente de trabajo y que esta relacionado por las relaciones informales y formales, por la personalidad de los miembros de la escuela y la dirección de la misma. Cid ,S. (2004) refiere que el ambiente organizacional es un factor de calidad en los centros educativos. Así mismo Muños Repiso (1995) pp.71 y Murillo, (1996), pp.66-72 , ratifica lo planteado anteriormente en cuanto a que es una de las características predictoras a la hora de medir la tasa de éxito académico, porcentaje de repetidores y satisfacción global de los miembros de la institución. Seria interesante evaluar, el impacto de este proceso en la calidad del proceso enseñanza aprendizaje, al influir tanto en la salud de los alumnos como en la de los docentes y en su ambiente organizacional.

\section{- La Participación del Docente}

Es uno de los grandes logros del proyecto, el cual está muy estrechamente relacionado con el punto anterior, es la participación entusiasta y responsable del docente tanto en su capacitación como en el desarrollo del proyecto. Para ilustrarlo se cita el testimonio extraído de dos grupos focales:

...Ya el docente no es pasivo, no, sino que ha abierto sus puertas, ha abierto los incentivos para que cada uno de los docentes, vaya más allá de la parte vista de contenido académico, sino más allá, del punto de vista de salud ...” (Preg.1 1:29; 236:241)....Yo sí creo que ha habido cambios muy importantes, los maestros no son los mismos, incluso la relación entre nosotros ha mejorado muchísimo ... (Preg.2 2:42; 664:667).

En otra escuela:

...Ya uno ha adquirido el compromiso con el proyecto, con la comunidad en general para mantenernos, como lo dice el mismo 
proyecto, saludables” (Preg. 1; 1:2; 33:36). ...ha creado en nosotros ese compromiso en verdad, esa responsabilidad, de seguirlo haciendo en forma continua...” (Preg. 1; 1:5; 51:58). ..

Este hecho es fundamental para la consolidación de las Escuelas Promotoras de Salud como lo plantea Gásperi (1998) al referirse a la importancia del rol del maestro en educación para la salud, y lo justifica por los siguientes factores: a) el docente dedica gran parte de su tiempo en cantidad y calidad, a la atención del niño y conoce sus problemas. b) Es una figura de referencia social aún en muchos espacios de la geografía venezolana, modelo a seguir por muchos niños. c) Es un organizador de métodos, estrategias educativas y conocimientos planificados por lo que moldea conductas e inculca aprendizajes permanentes en el educando. d) Posee comunicación directa con el niño y sus representantes.

Se identificaron con estas dos dimensiones una relación "triangular" entre el ambiente organizacional - participación del docente y otra que fue la identificación con el proyecto (ver figura 1).Esta relación se presentó en ambas direcciones, como influencia positiva o negativa, impulsando al docente a participar en un ambiente organizacional propicio, lo que asegura la identificación y apropiación del docente con el proyecto, fortaleciendo el trabajo mancomunado de gran parte de los docentes alrededor de una idea, objetivos y metas compartidas. De igual forma en las escuelas donde el ambiente organizacional no era el propicio, la participación no era suficiente y poco entusiasta, con poca identificación y compromiso por parte de los docentes. Por lo que inferimos que la forma en que se de esa relación, dependerá la sostenibilidad del proyecto, como efectivamente se constata en las respectivas escuelas con mas avances y logros con respecto a otras, de menos logros y poca participación, como se evidencia en la siguiente cita, en una escuela con dificultades para integrarse al proyecto:

...aquí se efectuó un taller, fueron más los del turno de la tarde que los del turno de la mañana... el proyecto sirve como para dos escuelas, funcionan dos escuelas, esa es la realidad, pero de hecho es una escuela...” (Preg, 1, 1:3; 31:35).

De la misma escuela se cita: . “...hay mucha apatía en muchos docentes, y esa apatía es la que no ha permitido que la escuela funcione como debe ser...” (Preg, 1, 1:9; 63:65). 

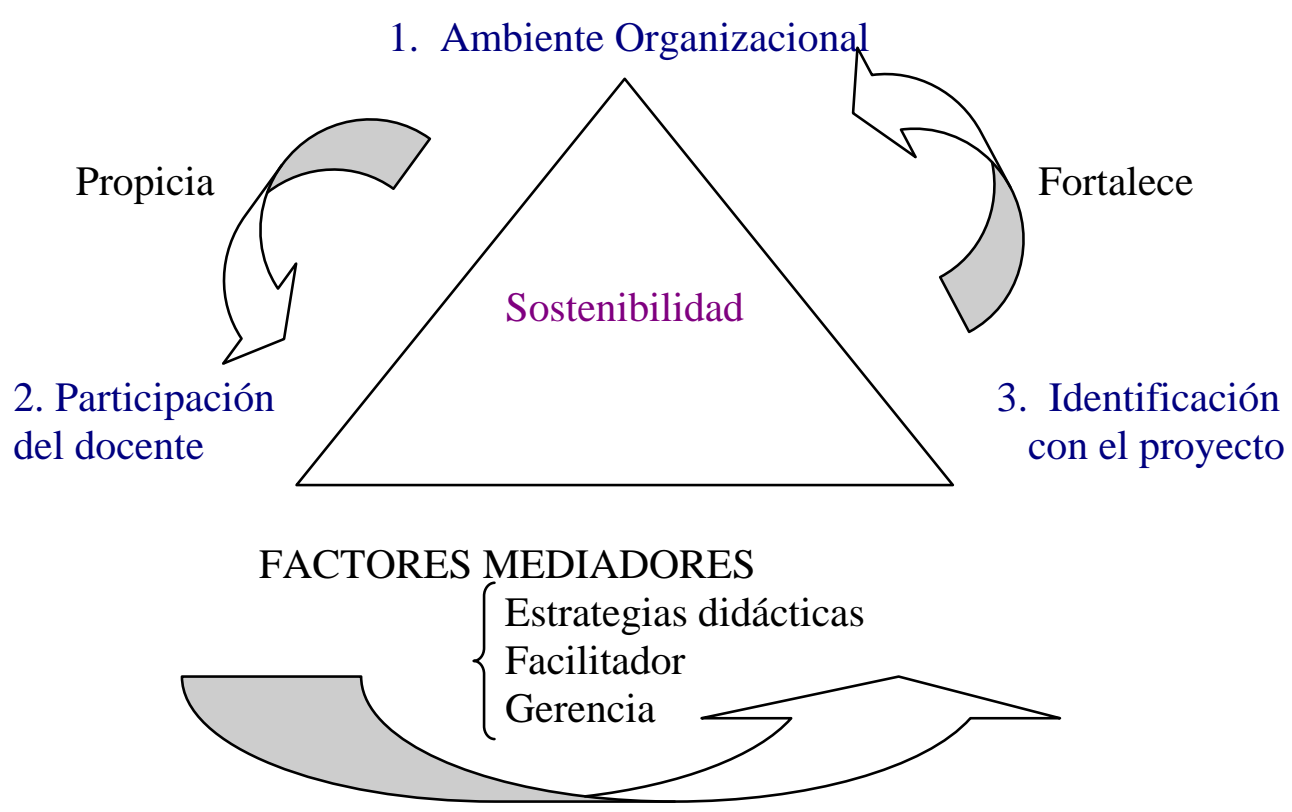

Asegura

Figura 1. Relación encontrada en los grupos focales entre la participación del docente , el ambiente organizacional de la escuelas y su identificación con el proyecto.

Como se aprecia en el grafico 1, además de la red conceptual que emergió alrededor de estas tres categorías citadas anteriormente, se encontraron factores mediadores para que el docente se interesara, se motivara a participar y lograra la identificación con el proyecto, estos factores son: las estrategias didácticas, las características del facilitador de los talleres y las actividades de planificación y coordinación por los gestores del proyecto. Al respecto, se citan sendos discursos

...En cuanto a la coordinación y a los que planifican, la selección del ponente, ya que debe estar bien identificado con el proyecto, que yo creo que la falla que hubo allí, que los dos facilitadores de esos dos talleres (donde hubo en varias escuelas algunos inconvenientes con dos facilitadores) no estaban bien identificados con lo que quería el proyecto en sí... (Preg, 4, 4:2; 57:66).

En otro, grupo focal se cita:

...fueron personas bien preparadas en el tema para abordar el tema, bien idóneos en la ejecución de las actividades, tenían, teníamos dinámicas grupales que eso incentivaba a todo el personal a participar fueron positivas..." (Preg, 4, 4:32; 371:375); ...aún los talleres me parecieron muy interactivos, ellos traían los temas, mas éramos nosotros quienes lo llevábamos al campo de trabajo... (Preg, 2, 2:29; 370:372). 
Siendo la participación del docente un punto importante en esta triple relación surgida en esta investigación ( ambiente organizacional- participación del docenteidentificación con el proyecto) y para reforzar lo expresado anteriormente, se cita a Hoy y Feldman (1999), quienes han trabajado sobre el mismo tema y afirman que una organización escolar sana, los profesores trabajan en forma cooperativa, con respeto mutuo y con altas expectativas para si mismo y para los estudiantes, así como sus directores gozan del respeto de los profesores y superiores y además pueden comunicarse e influir en el profesorado.

\section{- Aprendizaje de conceptos relacionados con el proyecto}

Otro gran logro ha sido el aprendizaje de conceptos relacionados con el proyecto, como: Salud, Promoción de Salud, Escuelas Promotoras de Salud, Rol del Docente, Visión y Misión de la escuela. El entender el paso del concepto biologicista, tradicional uní causal de salud por una concepción holística parcial (atención a problemas físicos y mentales) a uno más completo donde involucra un nuevo paradigma con una proyección hacia la promoción de una vida sana y de la sociogénesis multifactorial del proceso salud-enfermedad se considera un logro del proyecto. Se citan algunos fragmentos con el testimonio de estos aprendizajes:

...El concepto nuevo que veo dentro del proyecto es en primer término, la integración, es importante la integración de varios elementos para atacar un mismo problema... en el caso de la salud ..." (Preg.1 1:23; 238:241). “...Gran utilidad por cuanto se está trabajando con la parte preventiva, de repente nosotros desconocíamos de una forma conceptual, lo que era despistaje...” (Preg.1 1:34; 355:358).

En otra escuela ,se cita :

“...debe ser una salud vista desde varios factores, verdad, que inciden para que una persona pueda tener una buena salud en su entorno familia, entorno social, y el entorno escolar...” (Preg, 2, 2:4; 90:93); “...concepto de salud manejada como le dije ya a nivel preventivo, porque siempre conocemos la salud solo cuando estamos ya enfermos y vamos al médico y en este caso a nivel de prevención...” (Preg, 1, 1:39; 391:399).

Aquí es necesario precisar que no solamente los docentes adquirieron conocimientos nuevos, sino que pudieron darle operatividad y aplicación a temas que sólo manejaban a nivel teórico, como los referentes a Educación para la Salud: 
...Los conceptos nuevos, no es que hayan conceptos nuevos, sino que aprendimos a manejar conceptos que ya teníamos, porque habían cosas que ya nosotros conocíamos, pero no lo sabíamos manejar ... (Preg. 1 1:30; 304:307). “...Así como la Escuela Saludable ustedes dieron muchas cosas que ya nosotros sabíamos ...” (Preg.2 2:33; 463:465).

Por otra parte, otro logro aparte de entender estos conceptos tan importantes por personas formadoras de niños fue la aplicación de estos, no solo en la salud del niño sino a nivel personal, como veremos posteriormente.

\section{- Participación de la Comunidad Educativa}

Podemos añadir que en casi todas las escuelas, el proyecto ha animado directamente a los representantes a participar en las actividades de la escuela o indirectamente a través de los niños, en muchos casos, con diferentes grados de participación y con particularidades dependientes de las zonas y el tipo de población, consideramos la participación un logro del proyecto.

...uno ve la participación del representante, ahorita ve uno más al representante, un poquito más preocupado, va y le pregunta a uno mire maestra que podemos hacer con esto ...” (Preg. 1; 1:38; 438:441).

En otra escuela:

“...la misma asociación civil , en su presidenta que estuvo en el taller cuando hubo la presentación...observó y se motivó bastante, hasta nos compraron una cocina, un reverbero e hicimos que la Zona Educativa hasta nos diera unos implementos de cocina... y los viernes hacíamos comida en la escuela, con la ayuda de los padres y representantes, con los mismos niños, traían lo que podían, alimentos, se les pedía un alimento y cocinábamos ...” (Preg. 1; 1:41; 460:469).

En la opinión de los docentes y directivos, la participación de la comunidad educativa ha sido un factor determinante para la ejecución del proyecto, y aunque hay opiniones encontradas en las diferentes escuelas al respeto, en general podemos destacar a este aspecto como un logro del proyecto ya que en muchos casos se ha logrado involucrar a los padres y representantes en las actividades educativas de sus hijos y las de la escuela.

Cuando se evalúan proyectos de promoción de la salud, la participación es un parámetro primordial, para medir la efectividad de los proyectos y la sostenibilidad en los mismos, y en muchos casos se convierte en un factor predictor de la continuidad de 
los proyectos ya que es un proceso que propicia el análisis y priorización de necesidades, formula y negocia propuestas, haciéndolas socialmente factibles tal como lo plantea la OPS (1999), consideramos esto un valor agregado de este proyecto.

\section{- La utilidad en la salud del niño}

. Indudablemente fue reconocida por la mayoría de los docentes como uno de los aspectos de mayor utilidad del proyecto, según lo argumentado en varias escuelas: ...pero al principio del año escolar, la planilla para los despistajes, no se qué, siempre estamos hasta peleando por ella, este con el cuento de hacer el despistaje visual ... (Preg.3 3:25; 437:440).

...sobre el proceso de la vacuna que tengan los niños, este, que vacuna falta, de referir a los niños al ambulatorio, al menos solicitar al ambulatorio, para que venga a poner ciertas vacunas que hacen falta ..." (Preg.3 3:26; 444:450).

...todos los niños de la Escuela Pilade Montezuma se aprovecharon de las oportunidades, estaba la cooperativa y en la Alcaldía le pusieron lentes a todos los niños que lo necesitaban... el problema de caries el Dr. Agüero, en ese momento coordinador del Ambulatorio Carucieña se llevó un equipo portátil para resolver el problema allí mismo en la escuela ... (Preg.2 2:36; 589:595).

Como se desprende de estos testimonios, el proyecto ha resuelto problemas de salud en los niños, que en otra oportunidad no se hubieran podido resolver de forma masiva y oportuna.

\section{- Prestigio Institucional y mejor integración con el entorno}

En varias escuelas el proyecto sirvió para rescatar el valor y prestigio de la escuela ante la comunidad y ante los organismos dispensadores de salud, quienes en los actuales momentos ven a estas escuelas como aliados para mejorar la salud de los niños:

...han logrado integrarse con la propia comunidad, que para ellos era totalmente externa, o sea ellos han llegado a negociar y a interrelacionarse en mejores condiciones con la Iglesia y con todas las asociaciones que están alrededor para mejorar la escuela y su propio entorno, trabaja el ambulatorio, trabaja la comunidad y esto lo he percibido yo, a través de los talleres de ambiente que se han venido dando ... (Preg.2 2:21; 355:362). 
... como escuela, yo me presento como Escuela Saludable y tiene las puertas abiertas en cualquier institución, ya sea a nivel del ambulatorio, a nivel del hospital o a nivel de cualquier otro organismo, la misma Alcaldía, Gobernación y como que toman más consideración ese logro de una Escuela Saludable ...” (Preg.1 1:34; 387:392).

Este ultimo hallazgo encontrado, ratificado por la mayoría de las escuelas estudiadas, crea un foco de atracción hacia esta iniciativa como una vía, para mejorar las condiciones ambientales de la escuela, legitimar la escuela como centro socializador y cambiar situaciones como las encontradas por Espinoza ( 1996) en el estado Zulia, donde la escuela es una institución desvinculada de las comunidades donde se hallan insertas.

\section{- Beneficio Personal y Laboral del docente.}

En reiteradas ocasiones el docente ha referido el beneficio aportado por el proyecto para su desempeño laboral, al ofrecerles herramientas útiles para aplicar en su programa, que de manera oficial y obligatoria debe ofrecer a los alumnos. Asi lo refieren los docentes:

...cualquier docente, sea de cualquier etapa, primera o segunda etapa o en el preescolar, puede incluir en su proyecto de aula, estos conocimientos, nosotros siempre hemos trabajado salud dentro del aula, verdad, sólo que ahora está enriquecida por este apoyo tan integral ... (Preg.3 3:13; 184:189).

..porque si lo meten ellos su proyecto pedagógico de aula o de plantel, esto no es una carga, esto es parte del curriculum ... (Preg.2 2:34; 534:549).

De igual manera, como ya se comentó en la utilidad del proyecto, está el beneficio personal que estos talleres le han proporcionado al docente en su vida personal para desenvolverse día a día con los compañeros de trabajo, la familia y el resto de las personas.

...aprendimos muchos cosas, las cuales estamos poniendo en práctica tanto en el aula como en nuestro hogar, entonces sería muy bueno que a ese proyecto se le diera continuidad” (Preg. 2; 2:43; 571:574). ). “...se enfoca mucho dentro del área familia que se puede adoptar todo el proyecto de Escuela Saludable a lo que dentro del curriculum aparece como área familiar...” (Preg. 1; 1:45; 422:425).

En otra escuela, se menciona: 
...permitió también la integración de la comunidad, los niños, el docente con el ambulatorio, como decir, hubo lo que están diciendo mis compañeros y de utilidad bastante, no solamente aquí en la institución sino en mi hogar (Preg. 1; 1:50; 408:472).

\section{CONSIDERACIONES FINALES}

La Responsabilidad Social Universitaria, según la OEA, BID (2007) es una política de calidad ética del desempeño de la comunidad Universitaria a través de la gestión responsable de los impactos educativos, cognitivos, laborables y ambientales que las instituciones de educación superior generan, en un dialogo participativo con la sociedad para promover una nueva gestión social del conocimiento que contribuya a un desarrollo sostenible de las comunidades. La universidad es en la actualidad, la institución más apropiada para facilitar esa articulación, entre actividad científica y la sociedad civil. Este trabajo presenta los logros de un proyecto de vinculación social, que articula Universidad a un sector importante para el desarrollo del país como el sector educativo, y en donde como Universidad no responsabilizamos para que ese sector se apropie, utilice y comparta un saber acumulado en el área pedagógica y de salud a favor del bienestar de los niños y de la comunidad educativa. Este trabajo demostró que puede ser posible lo expresado anteriormente y la gran utilidad estas iniciativas institucionales, para acompañar la vida de las personas y sus comunidades educativas para crecer y desarrollarse, aportándoles herramientas para promocionar su salud y mejorar sus rendimiento académico y darles la oportunidad de conocer otras alternativas para vivir una existencia digna.

El método empleado en esta investigación fue el más adecuado para dar respuesta a los objetivos planteados, ya que nos permitió obtener una riqueza de datos, analizados en detalle y en profundad, que quizás por otro método no lo obtendríamos. Igualmente nos dejo un conociendo en las lecciones aprendidas del proyecto investigado, dentro de las que se mencionan la necesidad de implantar estrategias para concientizar en el docente en su formación profesional, el rol de promotor de la salud y promotor social, debidos a los incalculables beneficios que esto le proporcionaría a los niños y a la escuela, tal como se evidencio en algunos de los resultados encontrados en este trabajo. Igualmente aprendimos lo importante que es profundizar en el estudio del ambiente organizacional de las escuelas, específicamente en aquellos factores del clima 
organizacional, que puedan frenar o impulsar la participación del docente y de los representantes, en las actividades de promoción de la salud dentro de las escuelas, ya que la participación social es fundamental en la sostenibilidad de estos proyectos.

Finalmente, es importante destacar, la confluencia entre salud y educación que se dio en este trabajo en cuanto a su objeto y sujetos de investigación y que aunque la relación que existe entre salud y educación son múltiples e inseparables, quizás el elemento común mas importantes, es que ambas comparten las aspiraciones y objetivos de desarrollo humano contemplados en las políticas internacionales de educación para todos, Salud para todos, y las metas del milenio para el desarrollo, OPS (2003). Desde una perspectiva integral y amplia, salud y educación son a la vez fuente y prerrequisito del bienestar el desarrollo humano y la riqueza social, económica y espiritual de los individuos y los pueblos.

\section{REFERENCIAS}

Aguilar, M. Ander Egg E.(1992). Evaluación de Servicios y Programas Sociales. Buenos Aires: Editorial Lumen.

Alvarado, J.; Garrido, A. (1996). Psicología social aplicada. Madrid, España: Mc Graw Hill.

Cid Sabucedo ,A (2004) El clima organizacional como factor de calidad en los centros de educación secundaria de la provincia de Ourense. Su estudio desde la perspectiva de la Salud. Revista de investigación Educativa. Vol.22. pp.113144

Denzim N., Lincoln, Y. (2000). Handbook of qualitative research. $2^{\text {nd }}$ edition. New Park, Sage.

Espinosa, I. (1996). El educador y sus acciones comunitarias. Tesis de grado Universidad del Zulia. Maracaibo, Venezuela: Ediluz.

Gasperi,R. ( 1998). Diseño de un programa de capacitación sobre Promoción para la salud dirigido a docentes de la Primera etapa de la escuela básica, en el Municipio Iribarren. Tesis de grado Maestría salud publica. Universidad Centroccidental Lisandro Alvarado. Barquisimeto.

Glasser, G. (1997). The gronded theory.Disponible Disponible: www.groundedtheory.com/review/html. Consulta Abril 2006.

Greene, W.; Simon, B. (1988). Educación para la salud. México: McGraw Hill Interamericana. 
Hoy, W.K.( 1996) Educational Administration. Theory, research and practice.5ta edition. Londres Mc. Hill.

Ippólito- Shepard,J (2003) Fortalecimiento de la iniciativa regional de Escuelas promotoras de Salud. Estrategias y líneas de acción. Washintong.DC. Serie Promoción de la Salud. Nro. 4 pp18-25.

Lejarraga H..(2004) Desarrollo de niño en contexto. Paidos. Buenos Aires.

Martínez, M. (1999) Evaluación Cualitativa de Programas. Psicoprisma. Asociación Venezolana de Psicología Social.

Martínez, M. (2004). Ciencia y arte de la metodología cualitativa. México: Edit. Trillas Ministerio de Salud y desarrollo Social. (2002) Dirección de vigilancia Epidemiológica. Caracas. Boletín epidemiológico semanal.

Muños , Repiso.M et all. (1995) Calidad de la educación y eficacia escolar. Estudio sobre la gestión de los recursos humanos. Madrid .CIDE.

Murillo ,F,J. (1996)غ Son eficientes nuestras escuelas?. Cuadernos de pedagogía.246.pp 66-72.

OEA. BID.2007. Curso como enseñar ética capital social y desarrollo .Estrategia RSU. Modulo II. Responsabilidad Social Universitaria. Washington

Organización Panamericana de la Salud. (1995) Educación para la Salud en el Ámbito Escolar. Una Perspectiva Integral. Washington. Serie HSS/Silos-37.

Organización Panamericana de la Salud. ( 2007).Salud en las Américas. Publicación científica y técnica nro.622. Washington DC.

Organización Panamericana de la Salud. (1999) Taller de Evaluación de Municipios Saludables. Informe Final. Washington D.C. 2-6

Organización Panamericana de la Salud. (2003) Escuelas promotoras de La salud Fortalecimiento de la iniciativa regional. Washington. Serie promoción de la salud nro.14

Organización Panamericana de la Salud (2002) Resumen de memoria de la III reunión de la red Latinoamericana de Escuelas promotoras de Salud. Quito. Ecuador. Washington. DC .

Organización Panamericana de la Salud (2006).Herramienta de comunicación para el desarrollo de entornos saludables. Serie Paltex para ejecutores de programas de salud. nro.46. Washington. DC .

Valles, M. (1999). Técnicas cualitativas de investigación social. Reflexión metodológica y práctica profesional. Síntesis sociológica. Madrid.

Worchel, S., Cooper, J. (2002). Psicología Social. Interamericana. México: Thomson editores. 